\title{
Assessment of Thyroid Dysfunctions in Type 2 Diabetes Mellitus Patients in Surman, Western-Libya
}

\author{
Ashraf Ahmed Ahmed ${ }^{1}$, Siddig Bushra Mohamed ${ }^{2}$, Salih Abdelgadir Elmadi ${ }^{3}$, \\ Abdelkarim A. Abdorabo ${ }^{1}$, Ismail Mohamed Ismail ${ }^{4}$, Amar Mohamed Ismail ${ }^{4}$, * \\ ${ }^{1}$ Department of Clinical Chemistry, Faculty of Medical Laboratory Sciences, University of Al-Neelain, Khartoum, Sudan \\ ${ }^{2}$ Department of Medical Laboratory Sciences, Faculty of Medical Technology, Sabratha University, Surman, Libya \\ ${ }^{3}$ Department of Clinical Chemistry, Faculty of Medical Laboratory Sciences, The National Ribat University, Khartoum, Sudan \\ ${ }^{4}$ Department of Biochemistry and Molecular Biology, Faculty of Sciences and Technology, University of Al-Neelain, Khartoum, Sudan
}

Email address:

amarqqqu@yahoo.com (A. M. Ismail)

*Corresponding author

\section{To cite this article:}

Ashraf Ahmed Ahmed, Siddig Bushra Mohamed, Salih Abdelgadir Elmadi, Abdelkarim A. Abdorabo, Ismail Mohamed Ismail, Amar Mohamed Ismail. Assessment of Thyroid Dysfunctions in Type 2 Diabetes Mellitus Patients in Surman, Western-Libya. International Journal of Clinical and Experimental Medical Sciences. Vol. 3, No. 1, 2017, pp. 1-4. doi: 10.11648/j.ijcems.20170301.11

Received: February 19, 2016; Accepted: March 1, 2016; Published: April 18, 2017

\begin{abstract}
Diabetes mellitus (DM) and thyroid dysfunction (TD) are the two most common endocrine disorders in clinical practice. The study aim to find out the prevalence of thyroid disorders in type 2 diabetes mellitus patients. In a cross-sectional study 369 diagnosed type 2 DM patients, of which 203 are males and 166 are females, age reneged from (18 to 73) were enrolled, from July 2016 to July 2016. Their demographic data were obtained by through a questionnaire. Serum FT3, FT4, TSH and thyroid Antibodies (Tg-Ab, AM-Ab and TR-Ab) were measured using TOSOH, ELISA and enzymatic methods respectively. Out the 369 type 2 DM patients, of whom $9.5 \%$ are thyroid disorders, $2.3 \%$ have hypothyroidism, $5.0 \%$ subclinical hypothyroidism and 2.2\% hyperthyroidism. Thyroid disorders are more common in female's 16.5:1 fold. Compared with the control group, hypothyroid and subclinical hypothyroid DM patients have significant increase in TSH while significant decreases were observed in FT3 and FT4. TSH was significantly lower while FT3 and FT4 were significantly higher in hyperthyroidism DM patients. Moreover $23.3 \%$ of hypothyroidism has positive Tg-Ab, $23.3 \%$ has AM-Ab and $100 \%$ of hyperthyroidism has positive TR-Ab. The data suggested that, the prevalence of thyroid diseases is common in type $2 \mathrm{DM}$ Libyan patients. Autoantibodies are the main causes of thyroid dysfunction, therefore, could predict and increase risk of thyroid disease, especially in females.
\end{abstract}

Keywords: Type 2 Diabetes Mellitus, Thyroid Dysfunction, Autoantibodies, Libya

\section{Introduction}

Thyroid disorders are the most common endocrine dysfunctions. The total prevalence of these disorders estimated to be 200 million worldwide ${ }^{1}$. Diabetes mellitus is an important health problem affecting major population worldwide. It is associated with chronic hyperglycemia and disturbances of carbohydrate, lipid, and protein metabolism ${ }^{2 \text {, }}$ ${ }^{3}$. Thyroid diseases and diabetes mellitus are most common endocrinopathies encountered in clinical practice. Diabetes and thyroid disorders have been shown to mutually influence each other and an association between these two conditions has been reported in literature ${ }^{4}$. Thyroid dysfunction in diabetes mellitus manifests either as hypothyroidism (clinical or subclinical) or hyperthyroidism (clinical or subclinical) and is reflected in the circulating levels of thyroid hormones, namely tri-iodothyronine (T3), thyroxine (T4) and thyroid stimulating hormone (TSH) which might have an impact on blood glucose homeostasis ${ }^{5,}{ }^{6}$. Various complexes as biochemical, genetic, hormonal and pathophysiological mechanisms encountered in influences of the mixed endocrine and non-endocrine disorders involved in the mechanism ${ }^{7}$. Thyroid diseases affect approximately 10 to $15 \%$ of the patients with diabetes, whereas in non-diabetes, 
the prevalence is approximately $6 \%$ because patients with one organ-specific autoimmune disease are at risk of developing other autoimmune disorders. On one hand, thyroid hormones contribute to the regulation of carbohydrate metabolism and pancreatic function. And on the other hand, diabetes affects thyroid function tests to variable extents ${ }^{8}$. Thyroid disorders are the most common condition which affects the endocrine system second to diabetes mellitus. As a result, it is common for an individual to be affected by thyroid diseases and diabetes 9, 10, 11. The problems with interpretation of serum TSH, T4 and T3 have been recognized. Various studies reported that thyroid disorders vary according to age, genders, ethnic and geographical areas 12,13 . Therefore our study plan was to compare the FT3, FT4 and TSH levels in type 2 diabetic patients with respect to different age and gender groups in the population of Surman, Western, Libya.

\section{Materials and Methods}

A cross-sectional study was conducted in type 2 diabetes mellitus patients who attending the out patients clinic at Surman General Hospital, in Libya, from July 2015 to July 2016. Patients with other endocrine diseases and autoimmune were excluded. A (n 369) type 2 DM patients were included, (n 203) males and (n 166) females, age ranged from (18 to $73)$ years old. Venipuncture blood $(5 \mathrm{ml})$ was collected from each participant. Sera were obtained by centrifugation at $3000 \mathrm{rpm}$, and then kept at $-20^{\circ} \mathrm{C}$ till used.

\section{Ethical Consideration}

The ethical approved was taken from local authorities committee (Ministry of Health), and all patients were be informed by the aim of the study, samples and information were used anonymously.

\section{Methodology}

The laboratory investigations FT3, FT4 and TSH were measured using completive (for FT3 and FT4) and sandwich (for TSH) immunochemistry techniques in fully automated immunoassay analyzer (TOSOH). Microplate immunometric assay was used to estimate (Tg-Ab, AM-Ab and TR-Ab), absorbance of tests were measured using ELISA reader spectrophotometer (Techan). The quality assurance and assay accuracy were assured by using normal and pathological control sera.

\section{Statistical Analysis}

The Student's $t$-test and ANOVA were employed to compare differences between the means of continuous variables. Person's correlation was used to associate between study parameters and variables. Results expressed as Mean \pm SD and percentage. $P$-values $\leq 0.05$ was considered statistically significant. Data were analyzed by statistical package of social science (version 21.0; SPSS Inc.).

\section{Results}

The analyses of frequency showed that, out of 369 type DM patients, 35(9.5\%) have thyroid disorders, of which $5.0 \%$ was subclinical hypothyroidism followed by $2.3 \%$ hypothyroidism and $2.2 \%$ hyperthyroidism, presented in figure 1.

As we have shown by ANOVA analyses results, it was found that, both hypothyroidism and subclinical hypothyroidism have significantly lower FT3 and FT4 $(1.52 \pm 0.56, \quad 2.62 \pm 0.32$ and $0.49 \pm 0.24, \quad 0.90 \pm 0.12)$ in comparison with control group (Euthyroid) $(2.64 \pm 0.38$ and $1.80 \pm 0.24)$ respectively, with $p$-value $<0.05$, which presented in table 1.

Table 3 and 4 showed, the prevalence of autoantibodies among subtypes of thyroid dysfunction, out of 35 type 2 DM of which $7(23.3 \%)$ of hypothyroidism have positive $\mathrm{Tg}-\mathrm{Ab}$ and $7(23.3 \%)$ have positive AM-Ab. Moreover $5(14.3 \%)$ of hyperthyroidism have positive TR-Ab. All positive autoantibodies (TG-Ab, AM-Ab and TR-Ab) are found in females with $100 \%$.

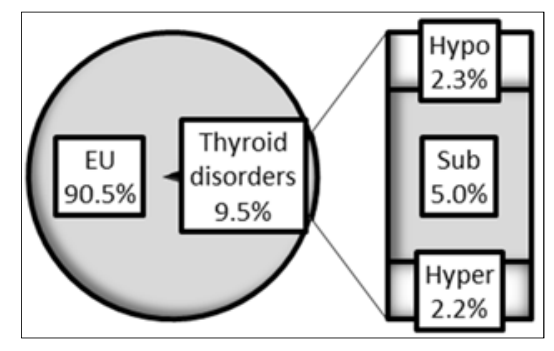

Figure 1. Frequency of thyroid disorders.

$\mathrm{Eu}=$ Euthyroid, $\mathrm{Hypo}=$ Hypothyroidism, Sub $=$ Subclinical hypothyroidism, hyper= Hyperthyroidism

Table 1. Comparison of mean thyroid hormones and FBG in different classes of thyroid disorders.

\begin{tabular}{|c|c|c|c|c|}
\hline Characteristics & FBG & FT3 & FT4 & TSH \\
\hline Control group (Euthyroid) & $212 \pm 70.5$ & $2.64 \pm 0.38$ & $1.80 \pm 0.24$ & $2.65 \pm 0.38$ \\
\hline Hypothyroidism & $\begin{array}{c}202 \pm 27.5 \\
* *\end{array}$ & $\begin{array}{c}1.52 \pm 0.56 \\
* *\end{array}$ & $\begin{array}{c}0.49 \pm 0.24 \\
* *\end{array}$ & $\begin{array}{c}7.63 \pm 3.70 \\
* *\end{array}$ \\
\hline Sub-hypothyroid & $\begin{array}{c}211 \pm 50.6 \\
* *\end{array}$ & $2.82 \pm 0.32$ & $\begin{array}{c}0.90 \pm 0.12 \\
*\end{array}$ & $\begin{array}{c}4.66 \pm 0.31 \\
* *\end{array}$ \\
\hline Hyperthyroidism & $\begin{array}{c}219 \pm 22.5 \\
*\end{array}$ & $\begin{array}{c}7.63 \pm 0.14 \\
* *\end{array}$ & $\begin{array}{c}9.43 \pm 6.88 \\
* *\end{array}$ & $\begin{array}{c}0.09 \pm 0.03 \\
* *\end{array}$ \\
\hline
\end{tabular}

* Significant, ** highly significant. Results expressed as Mean $\pm \mathrm{SD}$, significant differences considered as $\mathrm{p}$-value $\leq 0.05$ 
Table 2. Gender wise comparison of thyroid hormones and FBG.

\begin{tabular}{lccc}
\hline \multirow{2}{*}{ Characteristics } & Males & Females & \multirow{2}{*}{$\boldsymbol{P}$-values } \\
\cline { 2 - 3 } & Mean \pm SD & Mean \pm SD & 0.033 \\
FT3 $(\mathrm{pg} / \mathrm{ml})$ & $2.08 \pm 0.33$ & $2.83 \pm 0.13$ & 0.146 \\
FT4 $(\mathrm{ng} / \mathrm{dl})$ & $1.15 \pm 0.24$ & $1.42 \pm 0.20$ & 0.000 \\
TSH $(\mu \mathrm{IU} / \mathrm{ml})$ & $1.87 \pm 0.91$ & $2.57 \pm 0.16$ & 0.220 \\
FBG $\mathrm{mg} / \mathrm{dl}$ & $210 \pm 54.0$ & $218 \pm 82.0$ & \\
\hline
\end{tabular}

Results expressed as Mean $\pm \mathrm{SD}$, significant differences considered as p-value $\leq 0.05$

Table 3. Frequency of autoantibodies in different classes of thyroid disorders.

\begin{tabular}{|c|c|c|c|c|c|c|}
\hline \multirow{2}{*}{ Characteristics } & \multicolumn{2}{|c|}{ Tg-Ab } & \multicolumn{2}{|c|}{ AM-Ab } & \multicolumn{2}{|c|}{ TR-Ab } \\
\hline & $+\mathrm{ve}$ & -ve & $+\mathrm{ve}$ & -ve & $+\mathrm{ve}$ & -ve \\
\hline Hypothyroidism & $7(23.3 \%)$ & - & $7(23.3 \%)$ & - & - & - \\
\hline Subclinical Нуро & - & $23(65.7 \%)$ & - & $23(65.7)$ & - & $23(65.7 \%)$ \\
\hline Hyperthyroidism & - & - & - & - & $5(14.3 \%)$ & - \\
\hline
\end{tabular}

$+\mathrm{ve}=$ positive result $-\mathrm{ve}=$ negative result

Table 4. Frequency of autoantibodies in different classes of thyroid disorders among gender.

\begin{tabular}{|c|c|c|c|c|c|c|}
\hline \multirow{2}{*}{ Characteristics } & \multicolumn{2}{|c|}{ Tg-Ab } & \multicolumn{2}{|c|}{ AM-Ab } & \multicolumn{2}{|c|}{ TR-Ab } \\
\hline & Male & Female & Male & Female & Male & Female \\
\hline Control group (Euthyroid) & - & - & - & - & - & - \\
\hline Hypothyroid & - & $100 \%$ & - & $100 \%$ & - & - \\
\hline Sub-clinical hypo & - & - & - & - & - & - \\
\hline Hyperthyroid & - & - & - & - & - & $100 \%$ \\
\hline Total & $100 \%$ & $100 \%$ & - & $100 \%$ & - & $100 \%$ \\
\hline
\end{tabular}

\section{Discussion}

Insulin and thyroid hormones influence metabolism of carbohydrates, proteins, and lipids, hence their interrelation between DM and thyroid disorders ${ }^{7,14,15}$. Several studies have been published, investigated the association between diabetes and thyroid dysfunction. Therefore the present study carried out to evaluate the prevalence of thyroid disorders among type 2 DM in Libyan population, moreover to associate between thyroid disorders and autoantibodies.

The analyses of frequency showed that, the prevalence rate of thyroid disorders in type $2 \mathrm{DM}$ Libyan population was $9.5 \%, 5.0 \%$ is subclinical followed by $2.3 \%$ hypothyroidism and $2.2 \%$ hyperthyroidism. Thyroid disorders are more common in female than male 16.5:1 fold. In fact that, several epidemiological studies reported that, high prevalence of thyroid disorders (27.8\%) among type $2 \mathrm{DM}$ in India, and other study done in Spain reported $32.4 \%$ prevalence of thyroid disorders. These findings indicated that, type $2 \mathrm{DM}$ Libyan population has low prevalence of thyroid disorders in comparison with other populations.

Interestingly, the present study observed significant higher in TSH and FT3 among type 2 DM females in compression with males $p$-value $(0.000$ and 0.033$)$ respectively. Concurrent with previous finding that, estrogen hormone has direct role in thyroid follicular cells, and its role in thyroxine binding globulin (TBG) ${ }^{16}$, explain higher level of TSH and FT3 in type 2 DM females.

The present study provide evidence that, both hypothyroidism and subclinical hypothyroidism type 2 DM patients have significantly lower FT3 and FT4 level, while significantly higher was observed in mean TSH level when compared with control group (euthyroid). Concurrent with previous studies report, hypothyroidism is caused by insulin resistance, which led to less response of peripheral muscles to thyroid hormones ${ }^{17}$. On the other hand abnormality of thyroid hormones level attributed to insulin resistance, which decrease conversion of $\mathrm{T} 4$ to active $\mathrm{T} 3$, also reduced hypothalamus thyrotropin releasing hormone (TRH) in DM patients. In fact that, hypothyroidism result in lipid dysfunctions, so could magnify the cardiovascular disease and endothelial dysfunction in DM patients ${ }^{18}$.

Controversial finings have been documented regarding subclinical hypothyroidism, one reported an association between type $2 \mathrm{DM}$ and hypothyroidism, other conflicting findings $3,{ }^{17}$. Therefore further investigations are recommended. Concurrent with previous findings, thyroid dysfunctions most probably caused by autoimmunity and may be associated DM. Moreover thyroid dysfunction has greater impact on dyslipidemia and cardiovascular disease. Out of 35 of thyroid dysfunction patients, of whom 94.3 are female and 5.7 are male. TG-Ab and AM-Ab were found in $23.3 \%$ and $23.3 \%$ among hypothyroidism female respectively, while autoantibodies were not found in subclinical hypothyroidism. Moreover, all hyperthyroidism females have positive TR-Ab.

In addition several studies reported that, patients with specific autoimmune disease at higher risk of developing other autoimmune disorders, indeed that thyroid disorders are more common in female, interpreted higher thyroiditis among female. Similarity of several studies reported, thyroiditis is most common disorders in type 2 DM patients with hypothyroidism ${ }^{19}$. 


\section{Conclusion}

In conclusion, the results of present study suggest that, the prevalence of thyroid diseases is common in DM Libyan patients. Furthermore, autoantibodies are the main causes of thyroid dysfunction, therefore, could predict and increase risk of thyroid disease, especially in females. Meanwhile, routinely monitoring is recommended for diagnostic, prevention and management of related complications in type 2 DM Libyan population.

\section{Acknowledgements}

We express our sincere thanks to the director and laboratory staff of Surman General Hospital-Surman, Libya for their support and encouragements. Sincerely gratitude was extended to the technical staff in the department of clinical chemistry, Al-Neelain University-Sudan.

\section{Conflict of Interest}

The authors declare that no conflict of interest related to this manuscript.

\section{References}

[1] Rubina Mansoor, Syed Shakeel Raza Rizvi, Waseem Kausar, Faiza Aslam, Sibga Tul Huda, Comparison of TSH, T4 and T3 Levels in Primary Hypothyroidism in relation to gender and age in a Tertiary Care Hospital Ann. Innovative Journal of Medical and Health Science, 2011; 7 (4): 186-190.

[2] Makandar Asmabi, Sonagra Amit D, Biradar Shivaleela M, Quadri Shireen Swaliha, Effect of Thyroid Dysfunction on Metabolic Response in Type 2 Diabetic Patients. Unique Journal of Medical and Dental Sciences, 2015; 03 (01): 65-69.

[3] Ramesh V, Geetha R, Anitha D, Nrvk S, Thangarajan T. P. The Study of Thyroid Dysfunction among Type 2 Diabetic Patients, international Journal of Current Research and Academic Review, 2015; 3 (9): 14-18.

[4] Kiran Nagaraju, Amin Pirouz, Tayebeh Sadeghi, Pegah Esmaeili. Prevalence of thyroid dysfunction and its management in diabetic patients attending out patients clinic of Kim Hospital. International research journal of pharmacy, 2013, 4 (9): 132-35.

[5] Ray KK, Seshasai SR, Wijesuriya S, Sivakumaran R, Nethercott S, Preiss D, Erqou S, Sattar N. Effect of intensive control of glucose on cardiovascular outcomes and death in patients with diabetes mellitus: a metaanalysis of randomised controlled trials. The Lancet-Journal - Elsevier, 2009; 373 (9677): 1765-1772.

[6] Udoing CEJA, Udoh E, Etukudoh ME. Evaluation of thyroid function in diabetes mellitus in Calabar, Nigeria. Indian Journal Clinical of Biochemistry, 2007; 22 (2): 74-78.

[7] Singh G, GuptaV, Sharma AK, Gupta N. Evaluation of Thyroid Dysfunction Among type 2 diabetic Punjabi population. Advances Bioresearch journal 2011; 2 (2): 3-9.

[8] Padma V. and Anand N N. Prevalence of Thyroid Dysfunction in Type 2 Diabetic Patients. International Journal of Pharmacy Biochemistry Sciences, 2015; 6 (3): 289-294.

[9] Sudha J, Naved A, Prevalence of Thyroid Dysfunction in the Patients Visiting Tertiary Health Care Hospital, Faridabad; Haryana, International Journal of Scientific Research, 2013; 2 (10): 2277-8179.

[10] Akbar DH, Ahmed MM, Al-Mughales J. Thyroid dysfunction and thyroid autoimmunity in Saudi type 2 diabetics. Acta Diabetol journal, 2006; 43 (1): 14-18.

[11] Kadiyala R, Peter R, Okosieme OE. Thyroid dysfunction in patients with diabetes: clinical implications and screening strategies. International Journal of Clinical Practice 2010; 64 (8): 1130-1139.

[12] Kapadia K, Bhatt P, and Shah J. Association between altered thyroid state and insulin resistance. Journal of Pharmacology \& Pharmacotherapeutics, 2012; 3 (2): 156-160.

[13] Yang, G. R., Yang, J. K., Zhang, L., An, Y. H., Lu, J. K. 2010. Association between subclinical hypothyroidism and proliferative diabetic retinopathy in type 2 diabetic patients: a case control study. Tohoku Journal Experimental Medicine, 222 (4): 303-310.

[14] Athanasia P. B, Alexios S, Anthi K, Marina K, Petroula S, Stavros P, Prevalence of Thyroid Dysfunction Among Greek Type 2 Diabetic Patients Attending an Outpatient Clinic, Journal of Clinical Medical Research, 2010; 2 (2): 75-78.

[15] Akbar DH, Ahmed M. M, Al-Mughales J, Thyroid dysfunction and thyroid autoimmunity in Saudi type 2 diabetics. Acta Diabetologica, Acta Diabetol journal, 2006; 43 (1): 14-18.

[16] Ana Paula Santin and Tania weber Furlametto. Role of Estrogen in Thyroid Function and Growth regulation. Journal of Thyroid Research, 2011; 1-7.

[17] Chaoxun Wang. The Relationship between Type 2 Diabetes Mellitus and Related Thyroid Diseases, J Diabetes Res. 2013; $1-8$.

[18] Naveet Agrawwal, Manoj Gulati, Study of prevalence of thyroid dysfunction in patients with type 2 diabetes mellitus. International Journal of Contemporary medical research, 2016; 3 (8): 2212-2214.

[19] Manjunath SC, Krishnamurthy V, Puttaswamy BK. Prabhu S, Vishwanathaiah PM. Prevalence of subclinical thyroid disorders in type 2 diabetes mellitus. International Journal of Medical Public Health, 2013; 3: 330-334. 